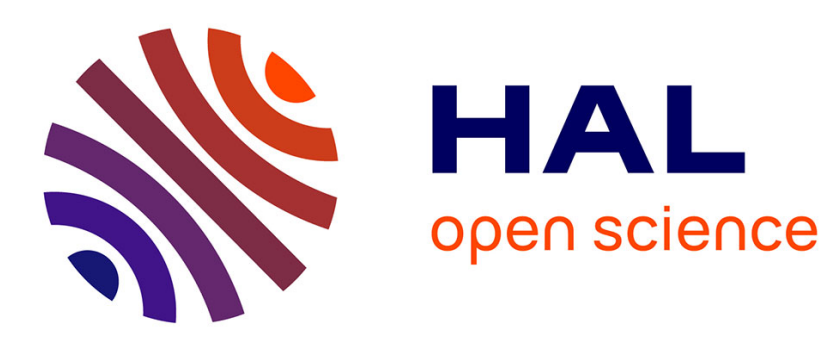

\title{
Identification of an Active Suspension in the Presence of Narrow Band Disturbances
}

\author{
Dan Stefanoiu, Ioan Doré Landau, Mihai Cornoiu
}

\section{To cite this version:}

Dan Stefanoiu, Ioan Doré Landau, Mihai Cornoiu. Identification of an Active Suspension in the Presence of Narrow Band Disturbances. MCPL 2010- 5th IFAC Conference on Management and Control of Production and Logistics, Sep 2010, Coimbra, Portugal. paper 63. hal-00712649

\section{HAL Id: hal-00712649 \\ https://hal.science/hal-00712649}

Submitted on 27 Jun 2012

HAL is a multi-disciplinary open access archive for the deposit and dissemination of scientific research documents, whether they are published or not. The documents may come from teaching and research institutions in France or abroad, or from public or private research centers.
L'archive ouverte pluridisciplinaire HAL, est destinée au dépôt et à la diffusion de documents scientifiques de niveau recherche, publiés ou non, émanant des établissements d'enseignement et de recherche français ou étrangers, des laboratoires publics ou privés. 


\title{
Identification of an Active Suspension in the Presence of Narrow Band Disturbances
}

\author{
Dan Stefanoiu*, Ioan D. Landau**, Mihai Cornoiu*** \\ *Dept. of Automatic Systems \& Computer Science, "Politehnica" University of Bucharest, \\ Splaiul Independentei no. 313, Bucharest - 060042, ROMANIA \\ (Tel: +40-21-316-9561; E-mail: danny@indinf.pub.ro). \\ **GIPSA Lab, Polytechnic National Institute of Grenoble, FRANCE \\ (E-mail: landau@gipsa-lab.grenoble-inp.fr) \\ ***Ph.D. student with the same affiliation as the first author \\ (E-mail: mihai.cornoiu@gmail.com).
}

\begin{abstract}
This paper aims to describe a comparative study regarding several identification models and control solutions for an active suspension system, while corrupted by narrow band disturbances. The tested identification models are linear and belong to ARMAX class. Several identification methods were compared in terms of disturbances rejection, both in open and closed loop cases. Beside the Least Squares class of methods, Minimum Prediction Error and Minimum Output Error methods have been investigated as well. The subsequent controller has been designed for the best found models and is either of RST or Q-parameterized class. After several simulations with real data, one has found that models estimated by means of Generalized Closed Loop Output Error (GCLOE) Method are seemingly the best ones.
\end{abstract}

\section{INTRODUCTION}

The active suspension system is already under study at many great automobile companies. This is the reason some research projects are developed in the academic filed as well. The GIPSA Laboratory from Grenoble (France) is hosting such a system, on both research and didactical purposes. Fig. 1 illustrates a picture of that active system.

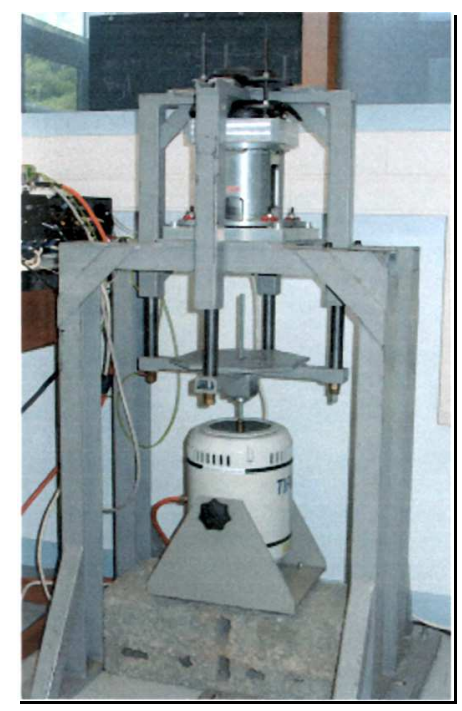

Fig. 1. Photo of the active suspension system hosted by GIPSA Laboratory from Grenoble (France).

Such a system is quite complex and mainly consists of two sub-systems: a primary one and a secondary one, as Fig. 2 displays. The primary sub-system is actually the classical passive suspension. Its input is the primary force (seen as disturbance), while the output is the first residual force, as returned by the elastomere cone. (Forces are measured by means of accelerometers.) The cone is only able to partially attenuate the primary force.

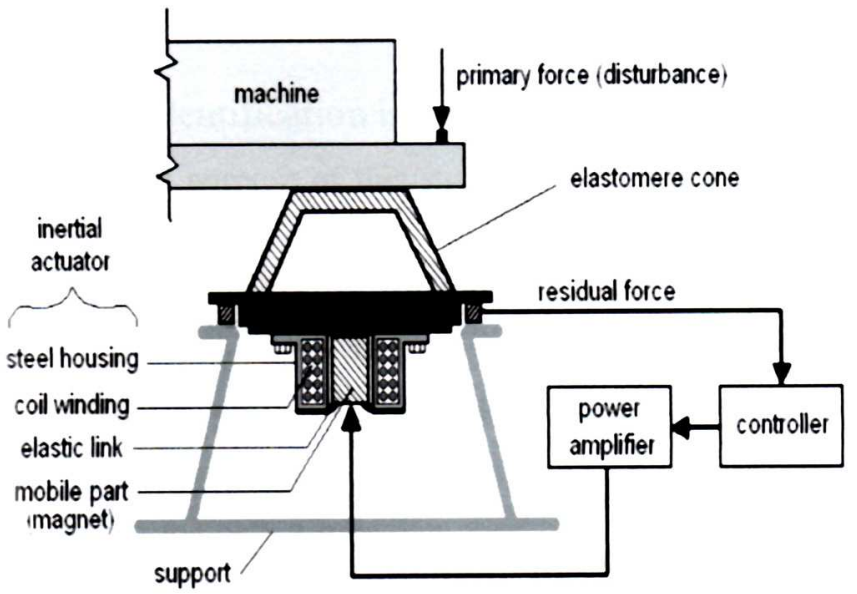

Fig. 2. Functional scheme of the active suspension system.

The secondary sub-system is the active extension of passive suspension by means of a controllable inertial actuator. This time, the input is the electrical power applied through the coil winding, in order to provide an attenuation force (the output), which is opposite with respect to the first residual force. By composing both outputs (the first residual force and the attenuation force), the second (final) residual force is obtained. Fig. 2 shows this resulted force only.

This paper focuses on the identification of the secondary path from active suspension, while working under the influence of narrow band disturbances of significant level. Subsequently, optimal controllers have been designed for the actuator, based on several identification models, in order to minimize the intensity of the final residual force. Since the signals between sub-systems are vibrations, the overall system exhibits a characteristic frequency of about $52 \mathrm{~Hz}$ and a critical resonance frequency of about $95 \mathrm{~Hz}$. Therefore, the study mainly focuses on the behaviour of active suspension excited by narrow band disturbances, which are centred at different 
frequencies, including the resonance one. (Further stochastic disturbances (seen as different coloured noises) are under consideration.) This topic has not been addressed in previous contributions (see for example [Landau et.al, 2005]), where identification has been performed in the absence of such disturbances. However, the narrow band disturbances may occur during the commissioning of the suspension system.

The article is structured as follows. The next section reviews some identification methods that have been employed in this study. (The ideal model is also emphasized.) Section 3 shortly describes the corresponding controllers. Section 4 is devoted to simulation results. Some concluding remarks and a succinct list of references complete the article.

\section{IDENTIFICATION OF SUSPENSION SYSTEM}

Here and hereafter, one assumes that the reader is accustomed with the terminology of System Identification and Digital Control Systems fields [Söderström-Stoica, 1989], [Landau, 1996], [Landau-Zito, 2005], [Stefanoiu et al., 2005]. Before reviewing the appropriate identification methods, one has to mention that the noiseless model of the suspension system has previously been identified by means of long Gaussian pseudo-random inputs, sampled at $800 \mathrm{~Hz}$. The model is of ARX type, with 14 zeros and 12 poles. In Fig. 3, the model spectrum has been drawn. One can notice the two peaks pointing to the characteristic (primary resonance) frequency $(51.66 \mathrm{~Hz})$ and the (secondary) resonance frequency $(\sim 95 \mathrm{~Hz})$, respectively. The performance of the noisy identification models will be assessed according to this ideal frequency characteristic.

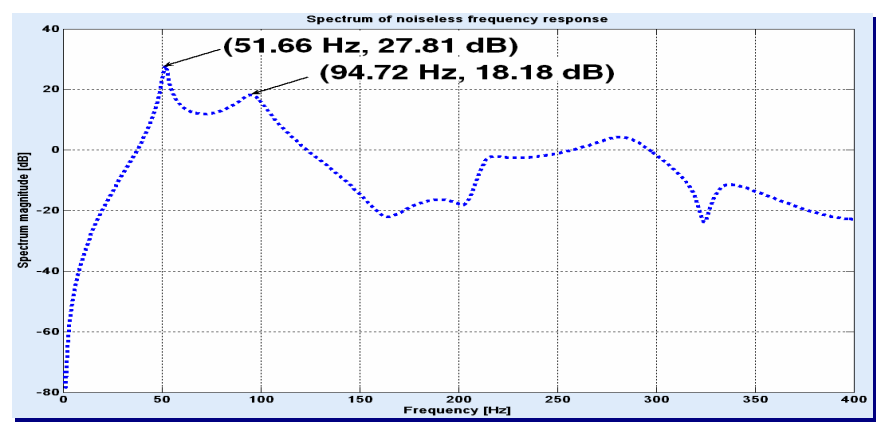

Fig. 3. Ideal spectrum of the active suspension system.

The ARX model actually belongs to ARMAX class, generally expressed by the linear regression equation below:

$$
y[n]=\boldsymbol{\varphi}^{\mathrm{T}}[n] \boldsymbol{\theta}+e[n], \quad \forall n \in \mathbb{N}^{*},
$$

where $\boldsymbol{\varphi}[n]$ is the regressors vector, $\boldsymbol{\theta}$ is the parameters vector (unknown) and $e$ is a (Gaussian) white noise. The two vectors have several compounds that match ARMAX class:

$$
\boldsymbol{\varphi}^{\mathrm{T}} \equiv\left[\begin{array}{lll}
-\boldsymbol{\varphi}_{y, n a}^{\mathrm{T}} & \boldsymbol{\varphi}_{u, n b}^{\mathrm{T}} & \boldsymbol{\varphi}_{e, n c}^{\mathrm{T}}
\end{array}\right], \quad \boldsymbol{\theta}^{\mathrm{T}} \equiv\left[\begin{array}{lll}
\boldsymbol{\theta}_{a}^{\mathrm{T}} & \boldsymbol{\theta}_{b}^{\mathrm{T}} & \boldsymbol{\theta}_{c}^{\mathrm{T}}
\end{array}\right],
$$

where $\boldsymbol{\varphi}_{x, m}^{\mathrm{T}}[n]=[x[n-1] \cdots x[n-m]], \forall n \in \mathbb{N}^{*}$, for some signal $x$ and structural index $m \in \mathbb{N}^{*}$, while $\boldsymbol{\theta}$ includes the coefficients of the 3 polynomials A, B and C. Obviously, $\boldsymbol{\varphi}$ consists of acquired and/or estimated data (since the white noise cannot be measured separately). The ideal ARX model only includes the first two compounds for each vector in (2).
Since the MA model of coloured noise could be non accurate, the ARMAX model has been extended to a more general Filtered Input Filtered Noise (FIFN) model. This time, the noise filter exhibits zeros as well as and poles. Thus the $\mathrm{C}$ polynomial of MA model is replaced by the couple of polynomials $\{C, D\}$. The FIFN regression equation is however similar to (1), but with a coloured noise $v$ instead of the white noise $e$. The noise $v$ is obtained by an AR model, with D polynomial playing the filtering role (all poles).

Two types of identification methods are bond to ARMAX/FIFN models (1) (of suspension system): open loop and closed loop. When the identification ignores the automatic controller, one can rely on the following methods [Dauphin-Tanguy et al., 2004]: (Recursive) Extended Least Squares (RELS), Open Loop Output Error with Extended Prediction Model (XOLOE), Generalized Least Squares (GLS). As of closed loop methods (when the controller is considered), the following were selected [Landau-Karimi, 1997]: Closed Loop Output Error (CLOE), CLOE with Extended Prediction Model (XCLOE), Generalized CLOE (GCLOE). A very brief description of the 6 above mentioned methods is presented next.

The RELS method actually employs a two stages LS strategy. The regressors vector from (2) is not entirely known, because $\boldsymbol{\varphi}_{e, n c}$ cannot be measured. Therefore, the first stage is aiming to identify an approximate ARX model, in order to estimate the noise with. Such a model has already been identified: the ideal one, with $n a=12$ and $n b=14$. Another model (with larger structural indexes) can also be identified from I/O data. Then the white noise can be estimated as the prediction error:

$$
\hat{e}[n]=\varepsilon[n]=y[n]-\boldsymbol{\varphi}_{\mathrm{ARX}}^{\mathrm{T}}[n] \hat{\boldsymbol{\theta}}_{\mathrm{ARX}}, \quad \forall n \in \mathbb{N}^{*},
$$

with natural notations. At the second stage, the estimates (3) are inserted in $\boldsymbol{\varphi}_{e, n c}$. Thus, $\boldsymbol{\varphi}_{e, n c}$ becomes $\boldsymbol{\varphi}_{\hat{e}, n c}$, whereas $\boldsymbol{\varphi}$ becomes $\hat{\boldsymbol{\varphi}}$. Now, the LS procedure is applied once more:

$$
\hat{\boldsymbol{\theta}}[N]=\left(\frac{1}{N} \sum_{n=1}^{N} \hat{\boldsymbol{\varphi}}[n] \hat{\boldsymbol{\varphi}}^{\mathrm{T}}[n]\right)^{-1}\left(\frac{1}{N} \sum_{n=1}^{N} \hat{\boldsymbol{\varphi}}[n] y[n]\right)
$$

where $N \in \mathbb{N}^{*}$ is the number of $\mathrm{I} / \mathrm{O}$ acquired data. The two stages above car recursively be repeated for increasing $N$.

The XOLOE method has small differences comparing to RELS. Its goal is to increase the convergence speed, by overcoming the bias between the true and estimated parameters faster. This time, $\mathrm{C}$ is replaced by $\mathrm{C}-\mathrm{A}$. The corresponding coefficients sequence $\delta=c-a$ is of length $n \delta=\max \{n a, n c\} . \quad$ In definitions (2), the following replacements are made: $\boldsymbol{\varphi}_{e, n c}$ by $\boldsymbol{\varphi}_{e, n \delta}, \boldsymbol{\theta}_{c}$ by $\boldsymbol{\theta}_{\delta}$ and $\boldsymbol{\varphi}_{y, n a}$ by $\boldsymbol{\varphi}_{\hat{y}, n a}$, where $\hat{y}[n]=\hat{\boldsymbol{\varphi}}^{\mathrm{T}}[n] \hat{\boldsymbol{\theta}}[n], \forall n \in \mathbb{N}^{*}$ is the noise free simulated output, with $\hat{\varphi}$ estimated exactly like in RELS. The difference $y-\hat{y}$ is actually the output error $(\underline{\mathrm{OE}})$, which has to be minimized by means of LS method. The OE formally replaces the prediction error $(\underline{\mathrm{PE}})$ computed like in definition (3) and creates the extended prediction model. The GLS method refers to the FIFN model (eventually with $\mathrm{C} \equiv 1$, i.e. without zeros) and can be implemented by means 
of the general Minimum Prediction Error (MPE), which is intrinsic recursive. The main problem is to estimate the coloured noise. This can be done by means of an approximate ARX model with long memory, like in (3), unless, now, not the white noise $e$ is estimated, but the coloured noise $v$. It follows that the noise filter is of ARMA type and can be identified through RELS method, from estimated output data.

The closed loop group of identification methods require the controller configuration. The configuration depicted in Fig. 4 is rather classical, of R-S-T type.

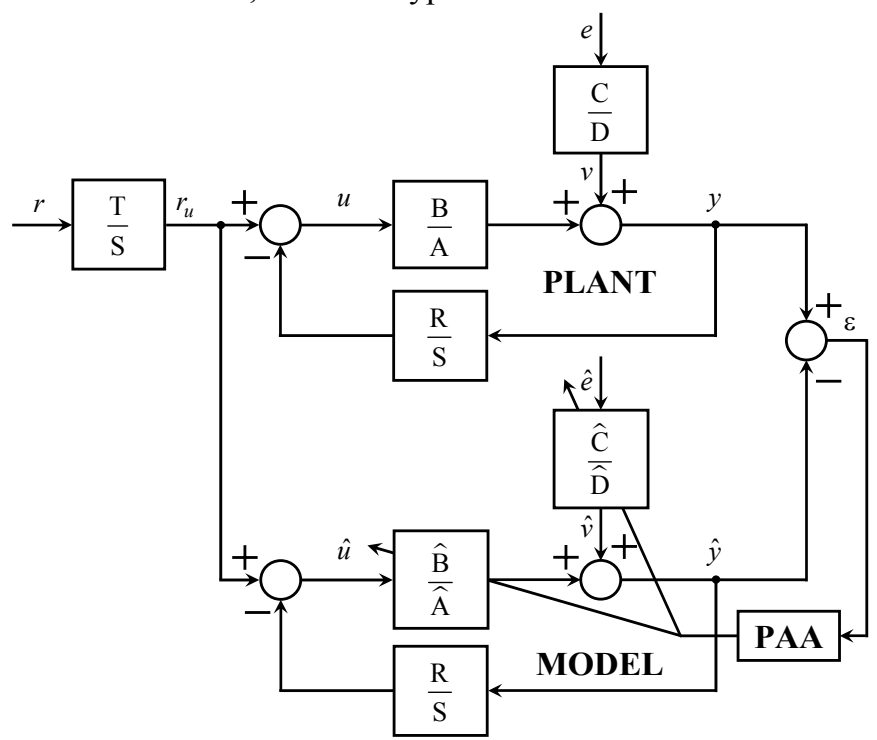

Fig. 4. Closed loop configuration with R-S-T controller.

Here, $\varepsilon$ plays the role of OE between the acquired output $y$ and the simulated output $\hat{y}$. The plant and the model include both filters (for input and for noise). However, the model can only work with estimated values of the white noise. The R-S-T controller depends on the identification model. It is designed such that noises are attenuated (or rejected) and the output verifies some performance criteria, according to reference $r$. Consequently, the $\boldsymbol{\varphi}_{u, n b}$ vector in (2) has to be computed/estimated by means of the controller, starting from the reference signal. Clearly:

$$
u[n]=\frac{\mathrm{T}\left(\mathrm{q}^{-1}\right)}{\mathrm{S}\left(\mathrm{q}^{-1}\right)} r[n]-\frac{\mathrm{R}\left(\mathrm{q}^{-1}\right)}{\mathrm{S}\left(\mathrm{q}^{-1}\right)} y[n], \quad \forall n \in \mathbb{N}^{*} .
$$

The PAA block stands for Parameter Adaptation Algorithm and performs model updating. (Note that the noise filter can be missing from the scheme, which decreases the controller performance.)

The CLOE method operates with the ARX model $(\mathrm{C} \equiv \mathrm{D} \equiv 1)$ and is based on minimisation of two types of OE: a priori $\left(\varepsilon_{0}\right)$ and a posteriori $\left(\varepsilon_{1}\right)$. They are defined by:

$\varepsilon_{0}[n]=y[n]-\hat{\boldsymbol{\varphi}}^{\mathrm{T}}[n] \hat{\boldsymbol{\theta}}[n] \& \varepsilon_{0}[n]=y[n]-\hat{\boldsymbol{\varphi}}^{\mathrm{T}}[n-1] \hat{\boldsymbol{\theta}}[n]$,

for any $n \in \mathbb{N}^{*}$. The rightmost terms of definitions (6) are referred to as the a priori output $\hat{y}_{0}$ and the a posteriori output $\hat{y}_{1}$, respectively. Ignoring the noises leads however to less accurate models, although the convergence is quite fast.
The XCLOE method approaches the ARMAX model ( $\mathrm{D} \equiv 1$ ) and operates with the closed loop predictor below:

$$
\hat{y}[n]=\left[1-\mathrm{A}\left(\mathrm{q}^{-1}\right)\right] y[n]+\mathrm{B}\left(\mathrm{q}^{-1}\right) u[n]+\frac{\mathrm{Q}\left(\mathrm{q}^{-1}\right)}{\mathrm{S}\left(\mathrm{q}^{-1}\right)} \varepsilon[n],
$$

for any $n \in \mathbb{N}^{*}$. In definition (7), the $\mathrm{Q}$ polynomial is:

$$
\mathrm{Q} \equiv 1+(\mathrm{C}-\mathrm{A}) \cdot \mathrm{S}-\mathrm{B} \cdot \mathrm{R} \text {. }
$$

Minimization of extended OE $y-\hat{y}$ (with $\hat{y}$ defined in (7)) is based on some RELS-like strategy.

The GCLOE method deals with the FIFN model, where both polynomials $\mathrm{C}$ and $\mathrm{D}$ can be non unit. The identification procedure is quite complex and, usually, makes use of MPE algorithm. This is the only method that fully employs the structure of Fig. 4.

Several other methods have been tested as well. But their performance was quite low, so they were removed.

\section{ON CONTROLLER DESIGN}

Two types of controllers have been adopted. The first one is already described in Fig. 4 and belongs to R-S-T class. This controller covers quite a large panoply of linear systems and can be designed starting from either open loop or closed loop identification models. The design procedure is largely described in various publications such as [Dauphin-Tanguy et $a l ., 2004$ ] or [Landau-Zito, 2005]. Before proceeding to the controller design, the user has to set some performance specifications. Usually, such specifications consists of the number and position of poles (real valued and/or complex conjugate pairs), together with their damping factors. For the active suspension system, poles are grouped in 3 categories: (a) two complex conjugate dominant poles that focus on the characteristic frequency $(51.66 \mathrm{~Hz})$, with a damping factor of 0.8 ; (b) four to six complex conjugate pairs of auxiliary poles that cover the frequency band $[90,400] \mathrm{Hz}$ with different damping factors (usually smaller than 0.8); (c) eleven to fifteen real valued auxiliary identical poles located on the interval $[0.22,0.4]$. The number and location of auxiliary poles depends on the identification model. In case of closed loop approach, updating of controller, on one side, and model estimation/adaptation, on the other side, are alternatively performed, through iterative procedures.

The second type of controller belongs to Q-parameterized class, also known as Youla-Kucera class [Blondel, 2006]. The corresponding identification and control scheme is drawn in Fig. 5. The controller aims to integrate a model of disturbances by means of $\mathrm{Q}$ polynomial. After few manipulations, from the scheme of Fig. 5, one obtains the noise-to-output equation:

$$
y \equiv \frac{\mathrm{A}\left(\mathrm{q}^{-1}\right)\left[\mathrm{S}_{0}\left(\mathrm{q}^{-1}\right)-\mathrm{B}\left(\mathrm{q}^{-1}\right) \mathrm{Q}\left(\mathrm{q}^{-1}\right)\right]}{\mathrm{A}\left(\mathrm{q}^{-1}\right) \mathrm{S}_{0}\left(\mathrm{q}^{-1}\right)+\mathrm{B}\left(\mathrm{q}^{-1}\right) \mathrm{R}_{0}\left(\mathrm{q}^{-1}\right)} \frac{\mathrm{D}\left(\mathrm{q}^{-1}\right)}{\mathrm{D}\left(\mathrm{q}^{-1}\right)} e .
$$

Eq. (9) implies that rejection of disturbances poles can be achieved with the following factorization (a Bézout-like equation, in fact):

$$
\mathrm{S}_{0}\left(\mathrm{q}^{-1}\right)-\mathrm{B}\left(\mathrm{q}^{-1}\right) \mathrm{Q}\left(\mathrm{q}^{-1}\right)=\mathrm{Q}_{0}\left(\mathrm{q}^{-1}\right) \mathrm{D}\left(\mathrm{q}^{-1}\right) \text {. }
$$




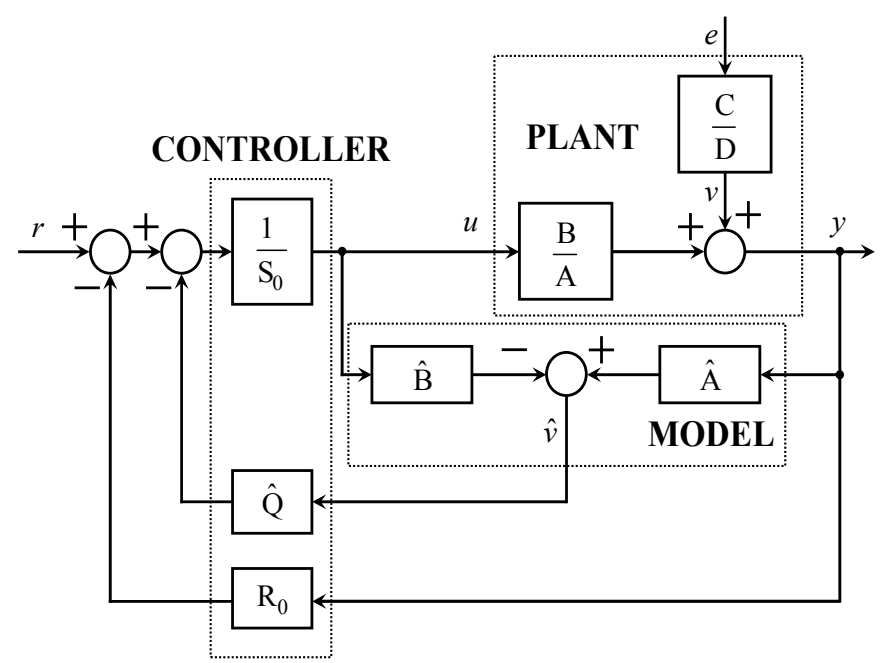

Fig. 5. Closed loop configuration with Youla-Kucera parameterized controller.

The unknown $\mathrm{Q}$ and $\mathrm{Q}_{0}$ polynomials are solutions equation (10). Similarly, the disturbances zeros can be removed by selecting $\mathrm{S}_{0}$ and $\mathrm{R}_{0}$ polynomials such that

$$
\mathrm{A}\left(\mathrm{q}^{-1}\right) \mathrm{S}_{0}\left(\mathrm{q}^{-1}\right)+\mathrm{B}\left(\mathrm{q}^{-1}\right) \mathrm{R}_{0}\left(\mathrm{q}^{-1}\right)=\mathrm{P}_{0}\left(\mathrm{q}^{-1}\right) \mathrm{C}\left(\mathrm{q}^{-1}\right)
$$

where the $\mathrm{P}_{0}$ polynomial has to be preset (by the controller design specifications). Notice that eq. (10) cannot be solved before eq. (11), since $\mathrm{S}_{0}$ needs to be known. Of course, both equations rely on the identification model. So, the controller has to be updated accordingly.

\section{SIMULATION RESULTS AND DISCUSSION}

The plant of Fig. 1 has been stimulated with 3 types of narrow band disturbances, at: $75 \mathrm{~Hz}, 90 \mathrm{~Hz}$ and $95 \mathrm{~Hz}$, respectively. The last one falls on the secondary resonance frequency range. The sampling frequency is $F_{s}=800 \mathrm{~Hz}$ and the I/O data block counts 10000 samples. The 6 identification methods provided valid identification models, as described in section 2. The structural indexes $n a, n b$ and $n c$ vary in range 10:16, whereas $n d$ is maximum 2 . The controllers were designed according to the principles of section 3. From the start, one has to say that both types of controllers led to very similar performance. However, the Q-parameterized controller is easier to implement.

To compare the models and especially to emphasize the open loop versus closed loop approaches, frequency characteristics of the identified main filter have been drawn, together with the ideal characteristic of Fig. 3. Also, the distance between each estimated frequency characteristic and the ideal one has been computed by means of error standard deviation (std), denoted by $\lambda$. More specifically, assume that $H$ is the ideal frequency characteristic (actually, the spectrum only), while $\hat{H}$ is one of its estimates, both expressed in dB. Normally, they are continuous maps. In order to draw their variations, let them uniformly be sampled. The number of samples, $K$, should be sufficiently large to have a good visual resolution (say at least 500). Then the std $\lambda$ (as distance between $H$ and $\hat{H}$ ) is computed as follows:

$$
\lambda=\sqrt{\frac{1}{K} \sum_{k=1}^{K}(H[k]-\hat{H}[k]-\langle H-\hat{H}\rangle)^{2}},
$$

where $H-\hat{H}$ is the error between the two characteristics, whereas $\langle H-\hat{H}\rangle$ is the average (the arithmetic mean) of the error. The smaller $\lambda$, the closer $H$ and $\hat{H}$ each other.

Figures 6, 7 and 8 illustrate the frequency characteristics of open loop models, for the three types of disturbances, with a resolution of $K=512$ samples. The red solid curve is the estimated characteristic $(\hat{H})$, while the blue doted curve shows the ideal characteristic $(H)$. For each couple of variations, the std $\lambda$ of error between the two characteristics was computed (like in eq. (12)). One can see that the LS methods perform differently than XOLOE, in terms of std. Whilst the LS methods perform the best at $75 \mathrm{~Hz}$ disturbance (i.e with minimum std), XOLOE improves as the disturbance approaches the secondary resonance frequency of $95 \mathrm{~Hz}$ (i.e. the std decreases). It seems that GLS is the very best open loop method (with the smallest std in all 3 cases). The best structural indexes for open loop models are: $n a=10$, $n b=12$ and $n c=12$, for ARMAX models identified with RELS or XOLOE methods; $n a=10, n b=12, n c=1$ and $n d=12$, for FIFN models identified with GLS method. The corresponded controller was of R-S-T type.

Figures 9, 10 and 11 focus on the results obtained with closed loop models and methods. The same graphical conventions and resolution like in case of open the loop approach are adopted, excepting for the solid curve, which turned in green. Apparently, the XCLOE method leads to the best models in terms of std $\lambda$. However, one can see that those models are not as accurate as the FIFN models estimated with GCLOE in 0-200 Hz subband (where disturbances lie). Therefore, one can consider that GCLOE is seemingly the best. This time, the optimal structural indices are: $n a=14, n b=14, n c=2$ and $n d=2$. The FIFN identification model also led to an efficient Youla-Kucera controller.

The performance of disturbances rejection is demonstrated in Figures 12 and 13. Both figures display the variations ranged on 2 columns. On the left side, the residual forces are drawn. On the right side, power spectral densities (psd) are depicted, with a graphical resolution of 512 samples (like within the previous figures). The dotted (blue) curve corresponds to psd of residual force when no controller is integrated in the active suspension system. The solid variations are actually the psd of residual forces from the left (red for open loop, green for closed loop models). Only the best models were considered, i.e. of FIFN type. The std of error between solid and dotted curves (denoted by $\sigma$, this time, but computed just like in eq. (12)) was also evaluated. In this context, as overall performance, the higher $\sigma$, the better disturbances rejection. However, the quality of disturbances rejection is not entirely revealed by $\sigma$. The narrow band of about $50 \mathrm{~Hz}$, centred on each disturbance peak (the dotted blue one in the figures), should be focused as well. Usually, when the disturbance attenuation is quite strong on the narrow band, some other disturbances outside the band could be amplified. This is the 
reason some identification models are only apparently better than others, when solely considering the overall std, $\sigma$. The rejection performance on the narrow band is assessed by $\sigma_{\mathrm{nb}}$, which is the std computed for the psd error restricted to the narrow band only. In general, $\sigma_{\mathrm{nb}}>\sigma$, since the number of psd samples is significantly smaller over the narrow band than over the whole band (the denominator in eq. (12) decreases).

Fig. 12 shows the performance of R-S-T controller when using FIFN-GLS (open loop) models. According to std $\sigma$, disturbances at $90 \mathrm{~Hz}$ are the least rejected, as Fig. 7 already predicted. However, the std $\sigma_{\mathrm{nb}}$ points to the disturbances at $95 \mathrm{~Hz}$ as the least rejected, which is a more accurate result. (Disturbances near the resonance frequency are harder to reject.) Fig. 13 illustrates the superior performance of Q-parameterized controller when using FIFN-GCLOE (closed loop) models, especially for 75 and $90 \mathrm{~Hz}$ disturbances. Again, the disturbances at $95 \mathrm{~Hz}$ are the least rejected. This time, both $\sigma$ and $\sigma_{\mathrm{nb}}$ lead to this conclusion.

\section{CONCLUDING REMARKS}

The case study developed in this paper concerned the identification and control of an active suspension system in the presence of narrow band disturbances at significant level. After testing several identification models and two types of controllers, one can say that the FIFN model identified by means of GLS or GCLOE methods allowed us to design the best controller in terms narrow band disturbances rejection. The Q-parameterized controller structure could be preferred to the R-S-T structure only for implementation purposes, because both types of controllers perform about the same. As future research, non linear identification models are under consideration, starting from the analytical system of equations that describe the active suspension dynamics.

\section{REFERENCES}

Blondel V. (2006). Simultaneous Stabilisation of Linear Systems, Springer Verlag, Heidelberg, Germany.

Dauphin-Tanguy G., Foulloy L., Popescu D. (2004). Modélisation, identification et commande des systèmes, Romanian Academy Press, Bucharest, Romania.

Landau I.D. (1996). Identification et commande des systèmes, Éditions Hermès, Paris, France.

Landau I.D., Constantinescu A., Rey D. (2005). Adaptive Narrow Band Disturbance Rejection Applied to an Active Suspention - An internal Model Principle Approach, Automatica, Vol. 41, pp. 563-574.

Landau I.D., Karimi A. (1997). Recursive Algorithms for Identification in Closed Loop: A Unified Approach and Evaluation, Automatica, Vol. 33, No. 8, pp. 1499-1523.

Landau I.D., Zito G. (2005). Digital Control Systems Design, Identification and Implementations, Springer Verlag, London, U.K.

Söderström T., Stoica P. (1989). System Identification, Prentice Hall, London, U.K.

Stefanoiu D., Culita J., Stoica P. (2005). A Foundation to System Identification and Modelling, Printech Press, Bucharest, Romania.

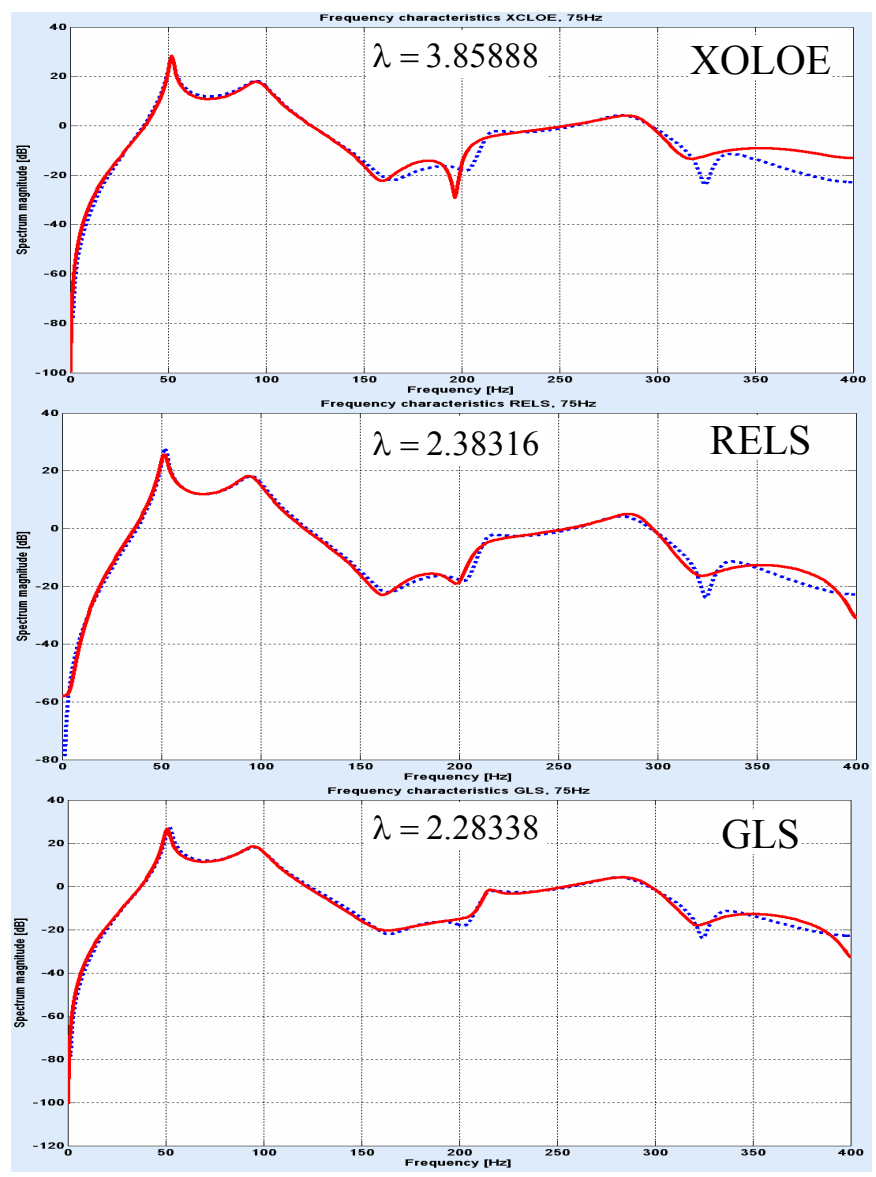

Fig. 6. Open loop models for $75 \mathrm{~Hz}$ disturbances.

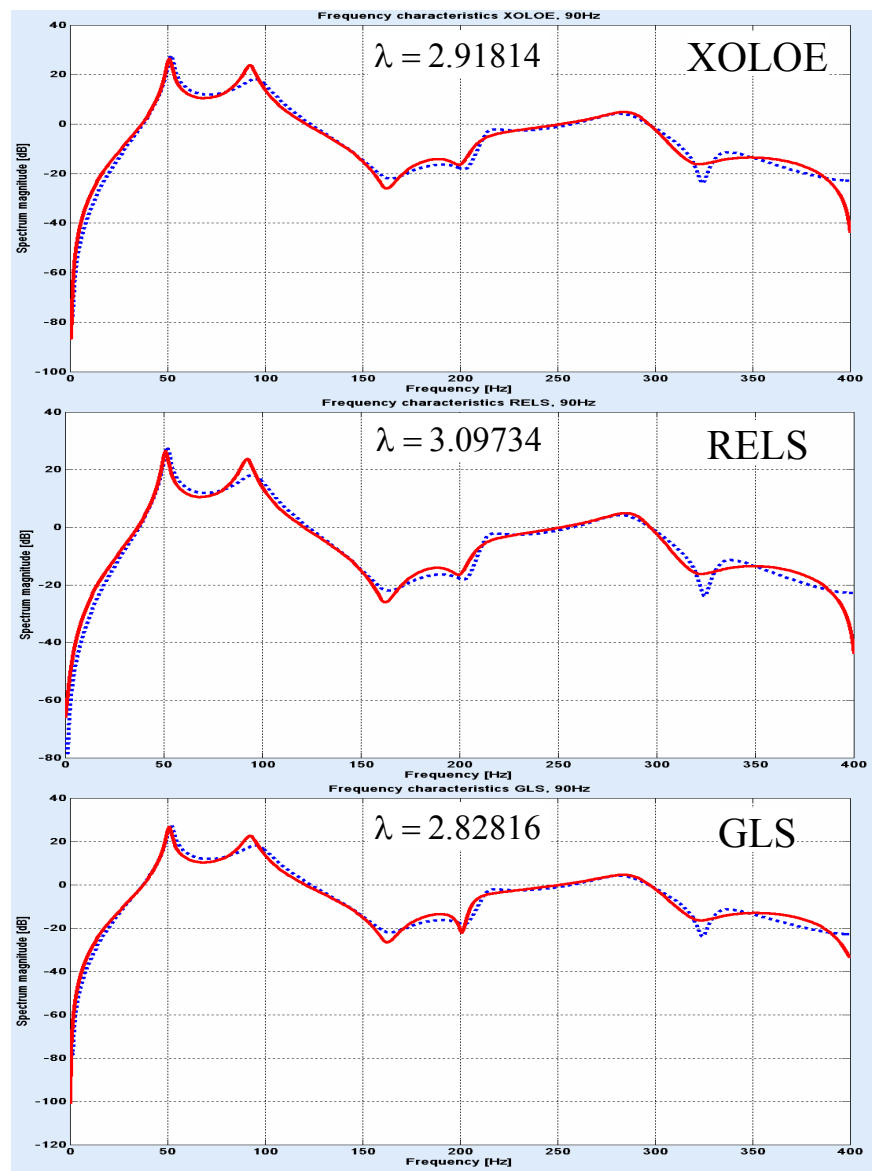

Fig. 7. Open loop models for $90 \mathrm{~Hz}$ disturbances. 


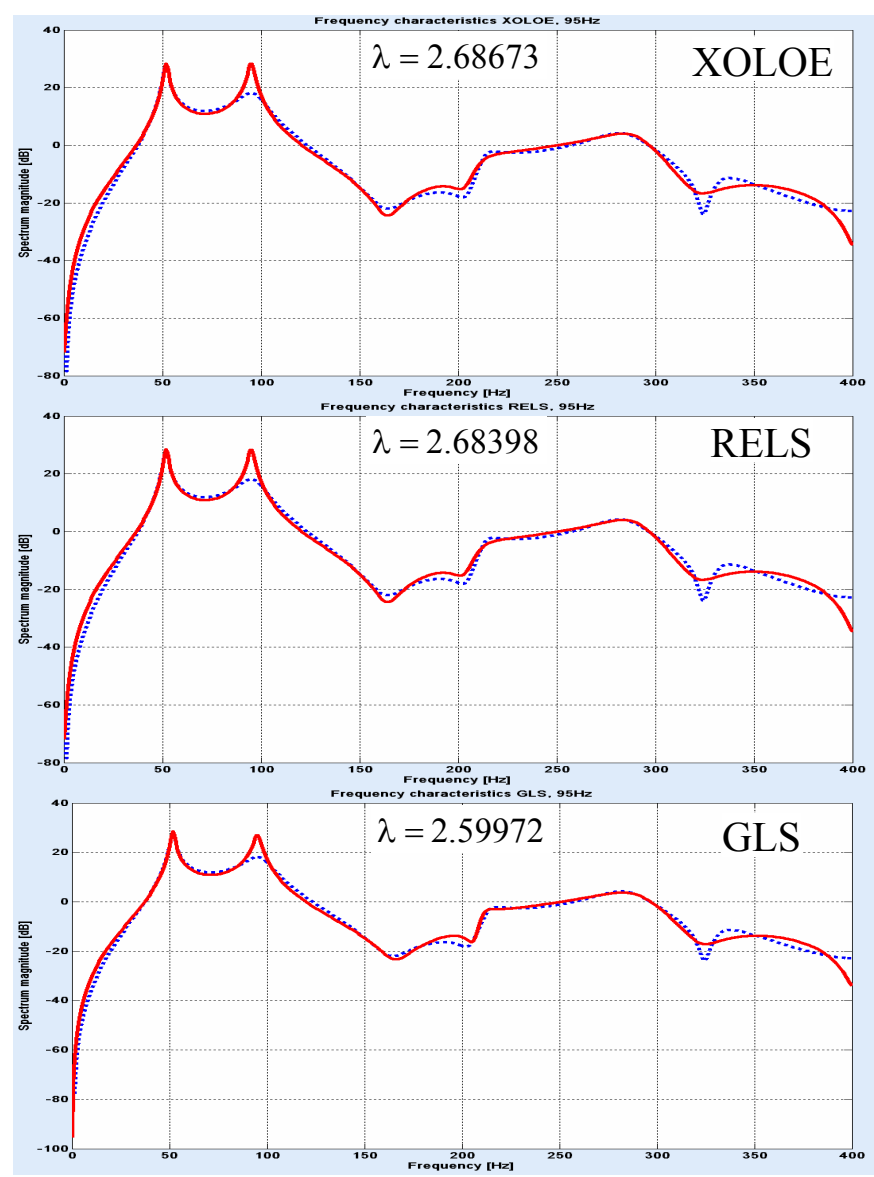

Fig. 8. Open loop models for $95 \mathrm{~Hz}$ disturbances.

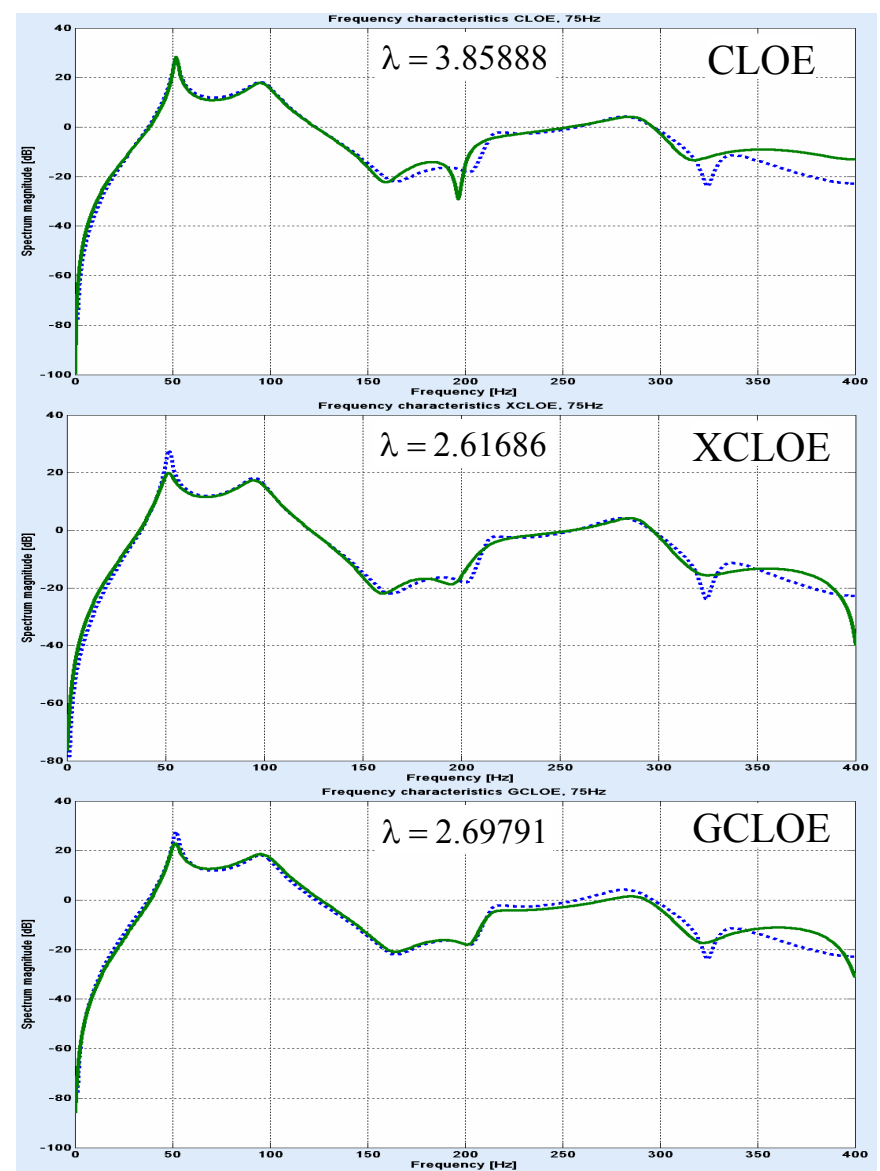

Fig. 9. Closed loop models for $75 \mathrm{~Hz}$ disturbances.

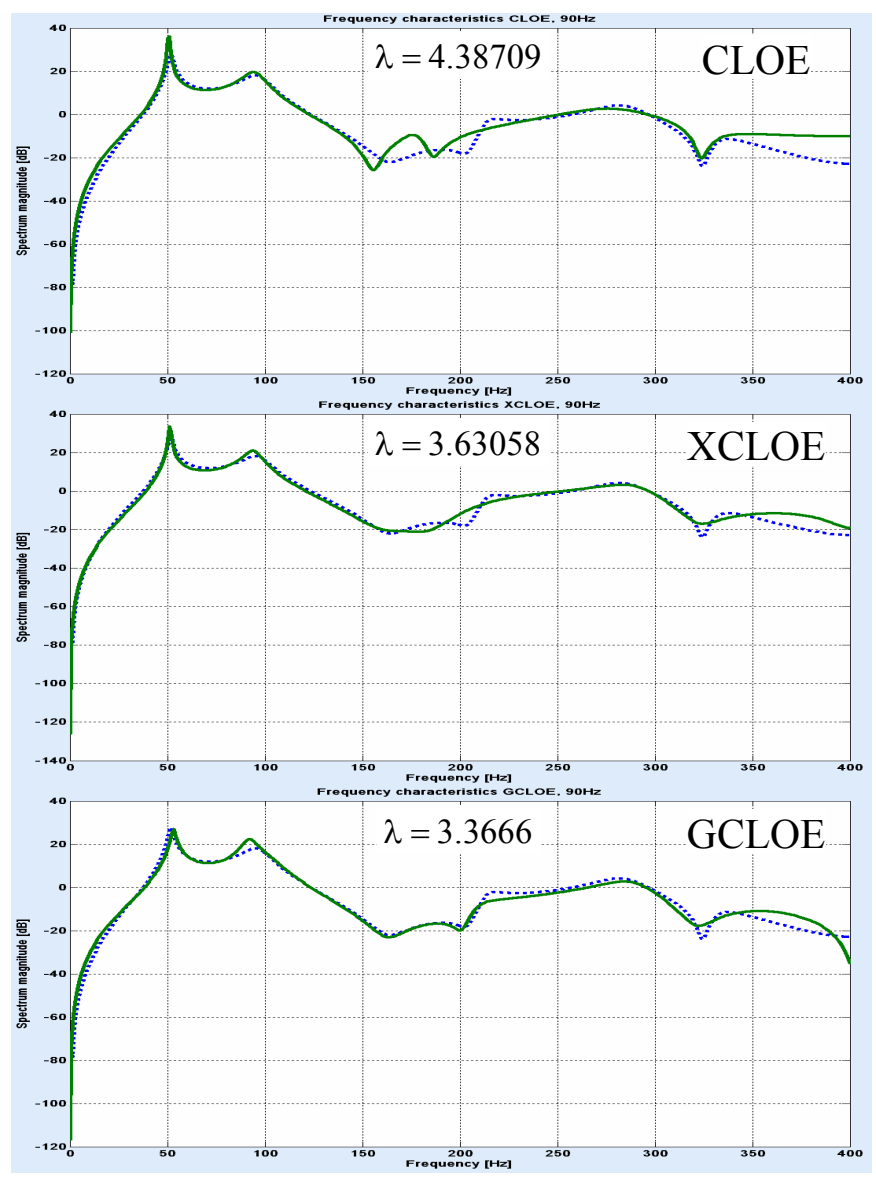

Fig. 10. Closed loop models for $90 \mathrm{~Hz}$ disturbances.

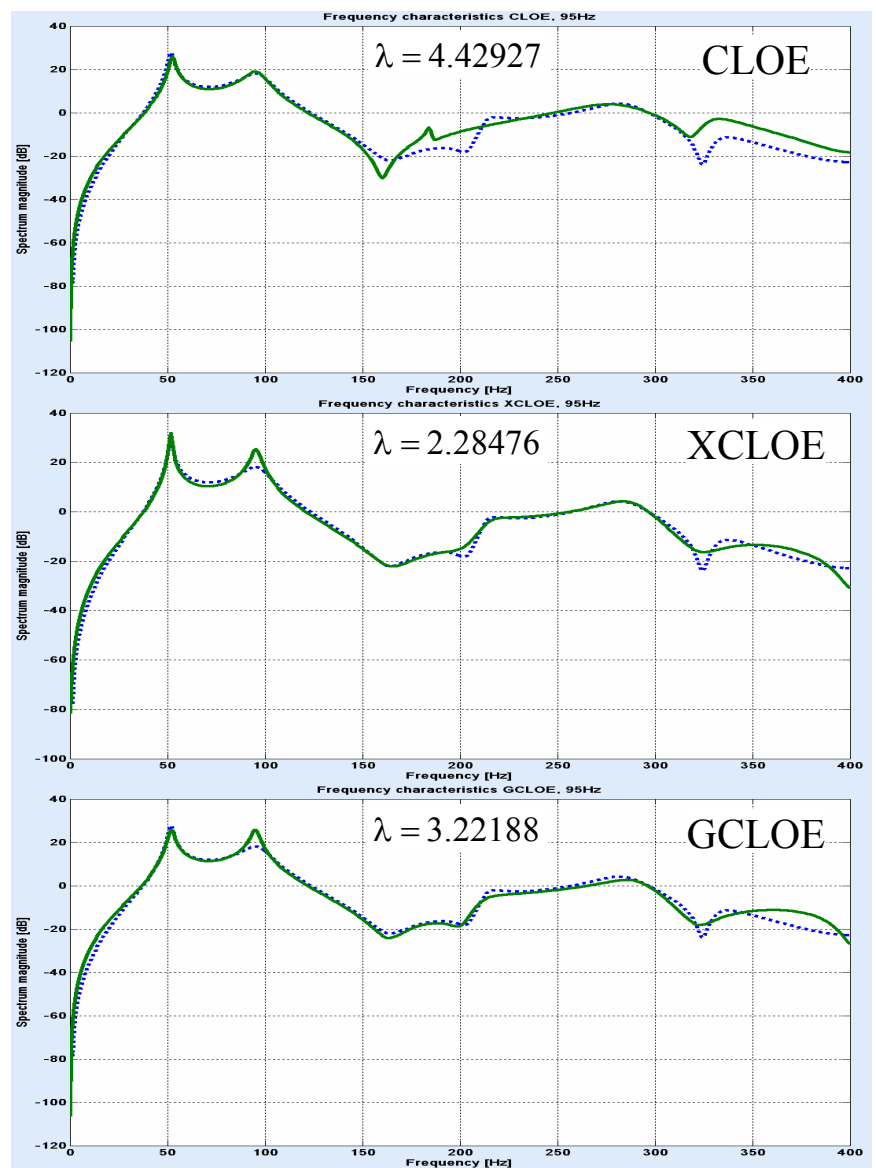

Fig. 11. Closed loop models for $95 \mathrm{~Hz}$ disturbances. 


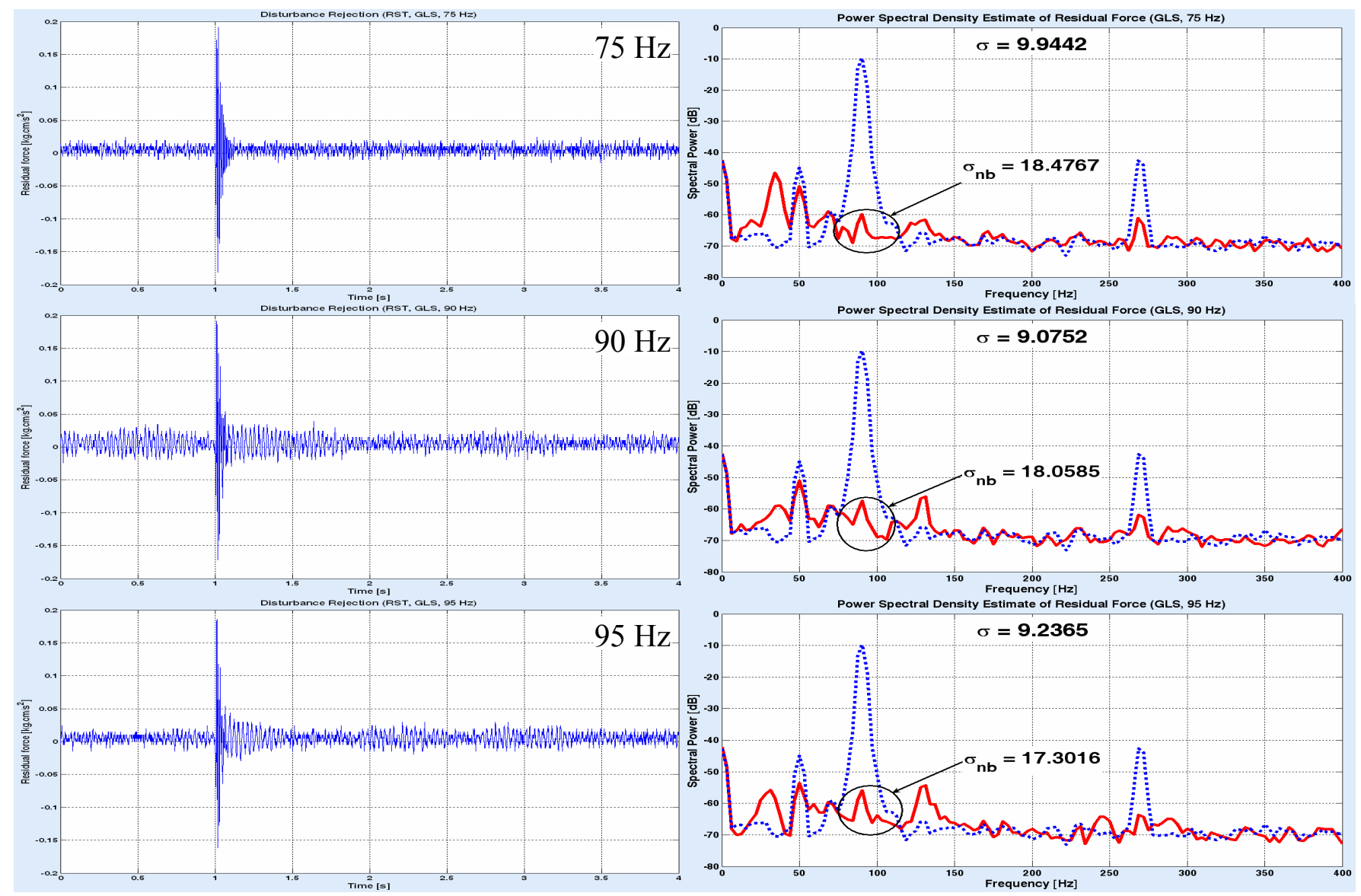

Fig. 12. Residual forces and spectra for FIFN-GLS models.

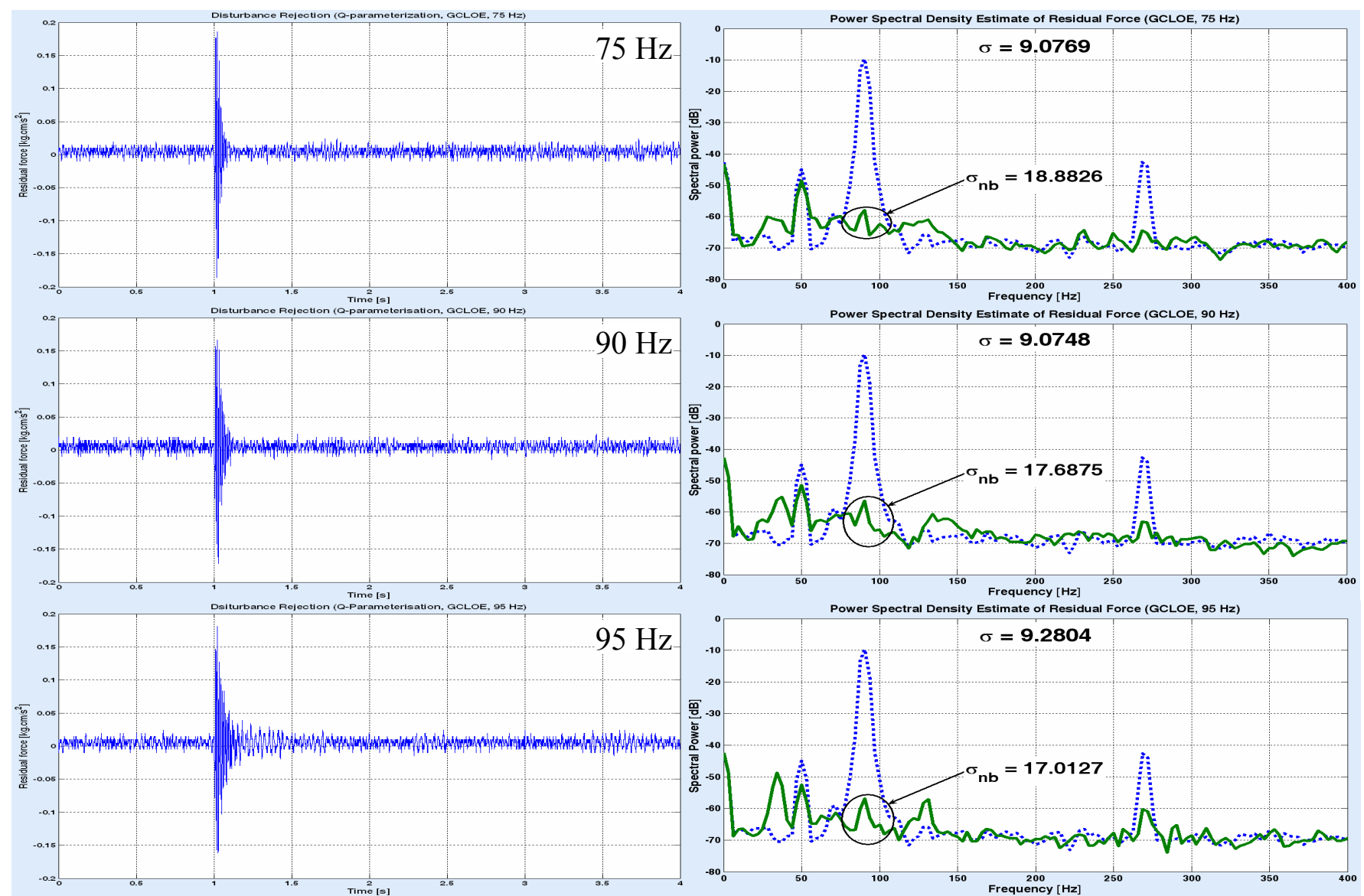

Fig. 13. Residual forces and spectra for FIFN-GCLOE models. 